\title{
Optical correlation based on the fractional Fourier transform
}

\author{
Sergio Granieri, Ricardo Arizaga, and Enrique E. Sicre
}

\begin{abstract}
Some properties of optical correlation based on the fractional Fourier transform are analyzed. For a particular set of fractional orders, a filter is obtained that becomes insensitive to scale variations of the object. An optical configuration is also proposed to carry out the fractional correlation in a flexible way, and some experimental results are shown. (c) 1997 Optical Society of America

Key words: Optical correlation, space-variant optical processing.
\end{abstract}

\section{Introduction}

Among several mathematical operations that can be optically implemented, correlation is one of the most important because it can be used for different applications, such as pattern recognition, aerial imagery, image feature extraction, object localization, etc. Correlation can be performed by use of the Fourier transform property of coherent optical systems, as, for example, with the VanderLugt $4 f$ configuration ${ }^{1}$ or the joint transform correlator. ${ }^{2}$ Since conventional correlation is a shift-invariant operation, the location of the correlator output simply moves if the object translates at the input plane. In many cases this property is necessary, but there are situations in which the position of the object provides an additional encoding feature, and so space invariance can be a drawback. In the search for space-variant optical filters, the fractional correlation,,$^{3,4}$ which is a generalization of the classical correlation operation that employs the fractional Fourier transform (FRT) instead of the conventional Fourier transform of the signals to be correlated, was recently proposed.

The FRT was defined mathematically by Namias ${ }^{5}$ and introduced to optics by Mendlovic et al. ${ }^{6-10}$ As it was analyzed in Ref. 9, the optical definition of the FRT can be established through a phase-space rotation of the Wigner distribution function. This point of view leads to the development of simple optical

The authors are with the Centro de Investigaciones Opticas (CIOp), C.C. 124, 1900 La Plata, Argentina. S. Granieri is also with the Facultad de Ciencias Exactas and R. Arizaga is also with the Facultad de Ingeniería, Universidad de La Plata, La Plata, Argentina.

Received 2 January 1997; revised manuscript received 2 April 1997.

0003-6935/97/266636-10\$10.00/0

(C) 1997 Optical Society of America devices for obtaining the FRT, but it also emphasizes the dual character of the FRT. Accordingly, the order $p$ varies from $p=0$ (pure spatial information) to $p=1$ (pure spectral information). Therefore, a filter that stores information about the FRT $(0<p<1)$ instead of the ordinary Fourier transform $(p=1)$ can be used to recognize objects with a space-variance degree that depends on the selected value of $p$.

The purpose of this paper is (i) to define the fractional correlation in a general way in which different orders of the FRT associated with the correlated signals are considered; (ii) to analyze some of its properties, as, for instance, those related to the spacevariance degree; and (iii) to demonstrate experimentally the fractional correlation that yields an application to build a filter insensitive to scale changes of the object. With respect to point (iii), a multiple-exposure hologram is employed to work in a parallel way. An optical configuration for performing the fractional correlation in a flexible way is also proposed and experimentally verified.

\section{Fractional Correlation}

For the following analysis the FRT is obtained by use of the optical setup proposed by Lohmann, ${ }^{9}$ which is shown in Fig. 1. In terms of the Fresnel diffraction, the amplitude distribution at the output plane corresponds to the FRT of order $p$ that is associated with the input signal $t\left(x_{0}\right)$, i.e.,

$$
\begin{aligned}
U p(x)= & \mathscr{F}^{(p)}\left\{t\left(x_{0}\right)\right\} \\
= & \exp \left(\frac{i \pi x^{2}}{\lambda f_{0} \tan \phi}\right) \int_{-\infty}^{\infty} t\left(x_{0}\right) \exp \left(\frac{i \pi x_{0}{ }^{2}}{\lambda f_{0} \tan \phi}\right) \\
& \times \exp \left(\frac{-2 \pi i x x_{0}}{\lambda f_{0} \sin \phi}\right) \mathrm{d} x_{0},
\end{aligned}
$$




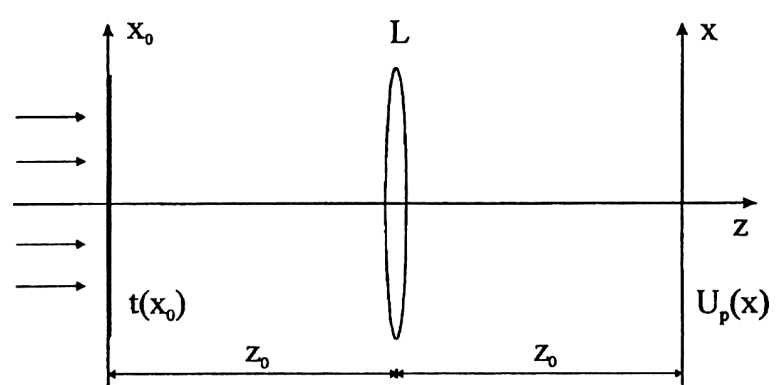

Fig. 1. Optical setup for performing the FRT of $t\left(x_{0}\right)$ for a given fractional order $p$, where $z_{0}=f_{0} \tan (p \pi / 4), f=f_{0} / \sin (p \pi / 2)$, and $f_{0}$ is a scaling factor.

where $\lambda$ is the wavelength, $f_{0}$ is a free-scale parameter, and $\phi=p \pi / 2$. For mathematical simplicity, we consider only one-dimensional functions. For a value of $p=1$, Eq. (1) reduces to the well-known Fourier transform relation. It should be noticed that Eq. (1) can be used as an optical definition of the FRT whenever the Fresnel approximation remains valid. Since the distance $z_{0}$ between the lens and the input (or output) plane is given by $z_{0}=f_{0} \tan (p \pi /$ 4 ), this limitation restricts the lower values of $p$ that can be chosen in Eq. (1). However, as we are interested in optical correlation applications for which the spectral information of the signal becomes relevant, the values of $p$ to be considered are large enough to be able to validate Eq. (1), i.e., $0 \ll p<1$.

The correlation of two functions $t_{1}(x)$ and $t_{2}(x)$ can be defined alternatively in the space or the spectral domain as

$$
\begin{aligned}
C_{12}(x) & =\int_{-\infty}^{\infty} t_{1}\left(x_{0}\right) t_{2}^{*}\left(x_{0}-x\right) \mathrm{d} x_{0} \\
& =\int_{-\infty}^{\infty} \tilde{t}_{1}(v) \tilde{t}_{2}^{*}(v) \exp (2 \pi i v x) \mathrm{d} v
\end{aligned}
$$

where $\tilde{t}(\nu)$ is the conventional ( $p=1$ ) Fourier transform of $t(x)$. By taking into account the second definition [Eq. (2b)] we propose the following expression as a generalized fractional correlation of $t_{1}(x)$ and $t_{2}(x)$ :

$$
C_{12}^{(p, q, r)}(x)=\mathscr{F}^{(q)}\left\{\mathscr{F}^{(q)}\left\{t_{1}\left(x_{0}\right)\right\} \mathscr{F}^{(r)} *\left\{t_{2}\left(x_{0}\right)\right\}\right\}
$$

Equation (3) becomes the standard correlation for the case of $p=-1, q=r=1$.

We next analyze the requirements to be fulfilled by Eq. (3) to get a meaningful fractional-correlation operation. The similarity condition [i.e., the same object in the same location: $\left.t_{1}\left(x_{0}\right)=t_{2}\left(x_{0}\right)\right]$ should maximize the correlation output for any value of the fractional orders. To derive an explicit expression for the fractional correlation, we replace the FRT operators in Eq. (3) by their equivalent definitions based on Fresnel integrals, as given by Eq. (1). Thus, we obtain

$$
\begin{aligned}
C_{12}^{(p, q, r)}(x)= & \exp \left(\frac{i \pi x^{2}}{\lambda f_{0} \tan \phi_{1}}\right) \int_{-\infty}^{\infty} t_{1}\left(x_{0}{ }^{\prime}\right) \exp \left(\frac{i \pi x_{0}{ }^{2}}{\lambda f_{0} \tan \phi_{2}}\right) \mathrm{d} x_{0}{ }^{\prime} \\
& \times \int_{-\infty}^{\infty} t_{2}{ }^{*}\left(x_{0}{ }^{\prime \prime}\right) \exp \left(-\frac{i \pi x_{0}{ }^{\prime 2}}{\lambda f_{0} \tan \phi_{3}}\right) \mathrm{d} x_{0}{ }^{\prime \prime} \\
& \times \int_{-\infty}^{\infty} \exp \left[\frac{i \pi x_{0}{ }^{2}}{\lambda f_{0}}\left(\frac{1}{\tan \phi_{1}}+\frac{1}{\tan \phi_{2}}-\frac{1}{\tan \phi_{3}}\right)\right] \\
& \times \exp \left[\frac{-2 \pi i x_{0}}{\lambda f_{0}}\left(\frac{x}{\sin \phi_{1}}+\frac{x_{0}{ }^{\prime}}{\sin \phi_{2}}-\frac{x_{0}{ }^{\prime \prime}}{\sin \phi_{3}}\right)\right] \mathrm{d} x_{0},
\end{aligned}
$$

where

$$
\phi_{1}=p \frac{\pi}{2}, \quad \phi_{2}=q \frac{\pi}{2}, \quad \phi_{3}=r \frac{\pi}{2} .
$$

For the purely spectral case $p=-1, q=r=1$ and Eq. (4) gives rise to a sharp correlation peak whenever $t_{1}\left(x_{0}\right)=t_{2}\left(x_{0}+\Delta\right)$, where $\Delta$ represents the shift between both correlated functions. For other values of the fractional orders Eq. (4) becomes sensitive to both the locations and the shapes of $t_{1}\left(x_{0}\right)$ and $t_{2}\left(x_{0}\right)$. However, the above-mentioned similarity condition, when applied to fractional correlation, requires that the quadratic phase exponential in the inner integral of Eq. (4), which generates a defocus effect, should be eliminated. This situation is achieved whenever the following relation for the fractional orders is satisfied:

$$
\begin{aligned}
& \frac{1}{\tan \left(p \frac{\pi}{2}\right)}+\frac{1}{\tan \left(q \frac{\pi}{2}\right)}-\frac{1}{\tan \left(r \frac{\pi}{2}\right)}=0, \\
& C_{12}^{(p, q, r)}(x)= \exp \left(\frac{i \pi x^{2}}{\lambda f_{0} \tan \phi_{1}}\right) \iint_{-\infty}^{\infty} t_{1}\left(x_{0}{ }^{\prime}\right) t_{2}{ }^{*}\left(x_{0}{ }^{\prime \prime}\right) \\
& \times \exp \left(\frac{i \pi x_{0}{ }^{\prime 2}}{\lambda f_{0} \tan \phi_{2}}\right) \exp \left(-\frac{i \pi x_{0}{ }^{\prime 2}}{\lambda f_{0} \tan \phi_{3}}\right) \\
& \times \delta\left(\frac{x}{\sin \phi_{1}}+\frac{x_{0}{ }^{\prime}}{\sin \phi_{2}}-\frac{x_{0}{ }^{\prime \prime}}{\sin \phi_{3}}\right) \mathrm{d} x_{0}{ }^{\prime} \mathrm{d} x_{0}{ }^{\prime \prime} .
\end{aligned}
$$

The fractional correlation as given by Eq. (7) is restricted to those cases in which the involved orders satisfy Eq. (6). For example, if $q=r$ then $p= \pm 1$, and Eq. (7) becomes the fractional correlation proposed and demonstrated experimentally in Refs. 3 and 4. In this case, Eq. (7) can be rewritten as

$$
\begin{aligned}
C_{12}^{(-1, q, q)}(x)= & \exp \left(\frac{-i \pi \sin (2 \phi) x^{2}}{2 \lambda f_{0}}\right) \int_{-\infty}^{\infty} t_{1}\left(x_{0}{ }^{\prime}\right) t_{2}{ }^{*} \\
& \times\left(x_{0}{ }^{\prime}-\sin \phi x\right) \exp \left(\frac{2 \pi i}{\lambda f_{0}} \cos \phi x x_{0}{ }^{\prime}\right) \mathrm{d} x_{0}{ }^{\prime},
\end{aligned}
$$

10 September 1997 / Vol. 36, No. 26 / APPLIED OPTICS 


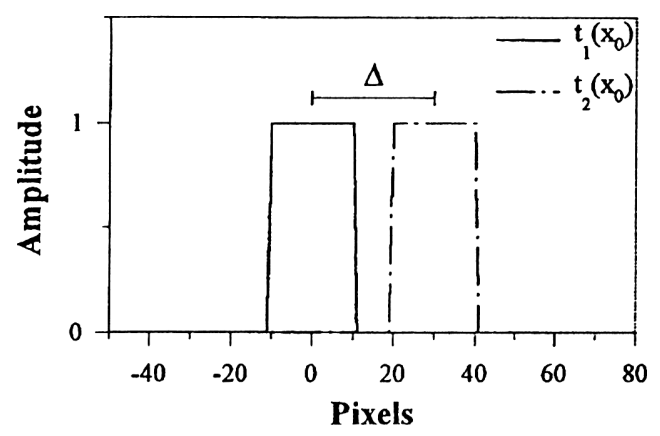

(a)

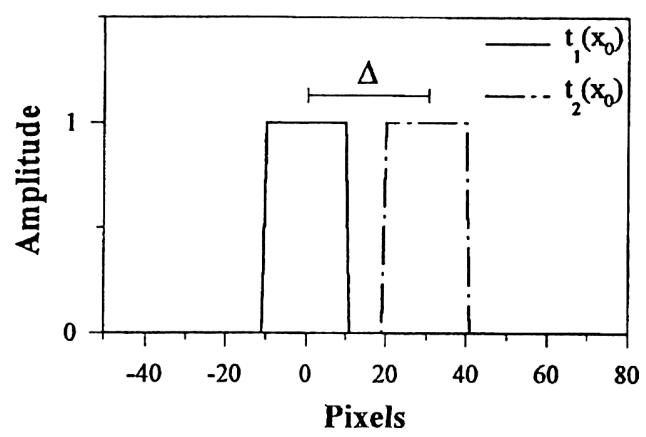

(a)
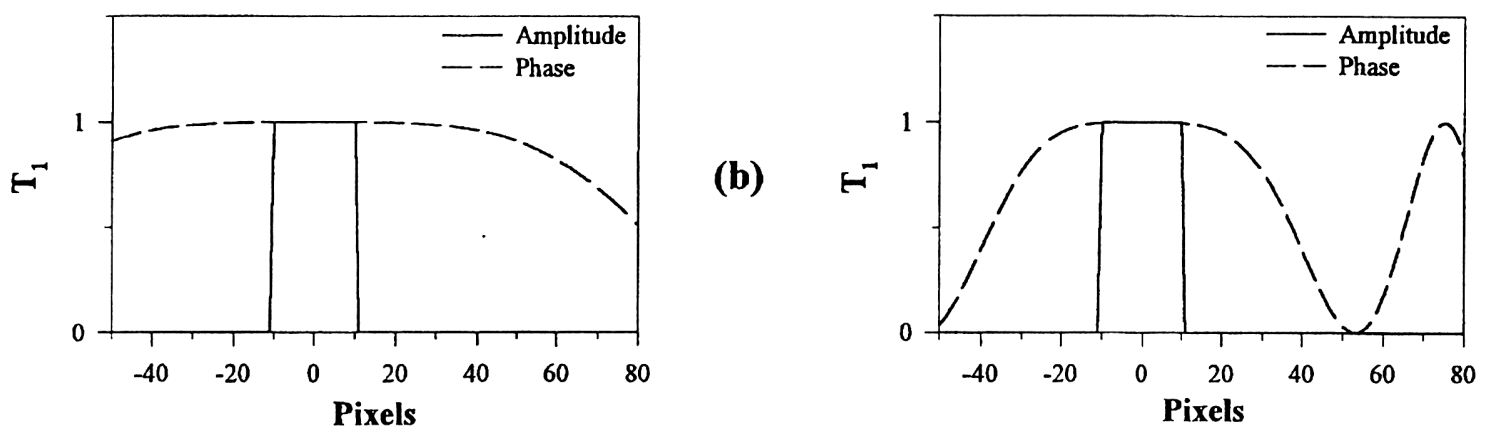

(b)
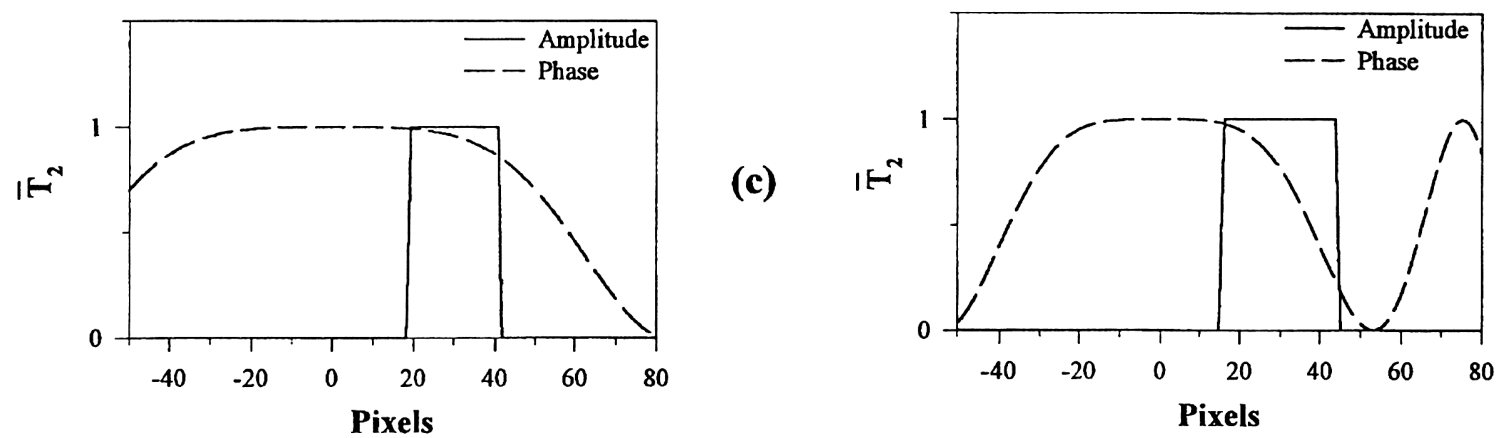

(c)

Fig. 2. (a) Object amplitude transmittances $t_{1}\left(x_{0}\right)$ and $t_{2}\left(x_{0}\right)$. (b) and (c) Modulus and phase of $T_{1}\left(x_{0} ; z_{1}\right)$ and $\bar{T}_{2}\left(x_{0} ; z_{2}\right)$, respectively, for $q=0.8$ and $r=0.7$.

or equivalently as

$$
\begin{aligned}
C_{12}^{(-1, q, q)}(x)= & \int_{-\infty}^{\infty} t_{1}\left(x^{\prime}+\frac{\sin \phi}{2} x\right) t_{2} *\left(x^{\prime}-\frac{\sin \phi}{2} x\right) \\
& \times \exp \left(\frac{2 \pi i}{\lambda f_{0}} \cos \phi x x^{\prime}\right) \mathrm{d} x^{\prime} \\
= & A_{t_{1} t_{2}}\left(y, \mu=\frac{-y}{\lambda f_{0} \tan \phi}\right),
\end{aligned}
$$

where $\phi=(\pi / 2) q, y=\sin \phi x$ and $A_{t_{1} t_{2}}(y, \mu)$ is the cross-ambiguity function, ${ }^{11}$ which is a dual phasespace representation coordinate-spatial frequency of the functions $t_{1}\left(x_{0}\right)$ and $t_{2}\left(x_{0}\right)$. Thus, the scaled fractional correlation for the several values of $q$ can be found from different slices in the phase-space $(y, \mu)$.

Fig. 3. (a) Object amplitude transmittances $t_{1}\left(x_{0}\right)$ and $t_{2}\left(x_{0}\right)$. (b) and (c) Modulus and phase of $T_{1}\left(x_{0} ; z_{1}\right)$ and $\bar{T}_{2}\left(x_{0} ; z_{2}\right)$, respectively, for $q=0.6$ and $r=0.4$.

This result was pointed out in Ref. 12, where it was analyzed by means of the analogy of optical spatial filters based on the FRT to wavelet transforms and adaptive neural networks. Besides, Eq. (9) means that all fractional correlations, from $q=0$ (vertical line, $y=0$ ) to $q=1$ (horizontal line, $\mu=0$ ) are contained in a single picture display of the ambiguity function.

For the more general case of $q \neq r$, the fractional correlation obtained from Eq. (7) can be rewritten as

$$
\begin{aligned}
C_{12}^{(p, q, r)}(x)= & \exp \left(\frac{i \pi x^{2}}{\lambda f_{0} \tan \phi_{1}}\right) \int_{-\infty}^{\infty} T_{1}\left(x_{0}{ }^{\prime} ; z_{1}\right) \\
& \times \bar{T}_{2}{ }^{*}\left(x_{0}{ }^{\prime}+\frac{\sin \phi_{2}}{\sin \phi_{1}} x ; z_{2}\right) \mathrm{d} x_{0}{ }^{\prime},
\end{aligned}
$$




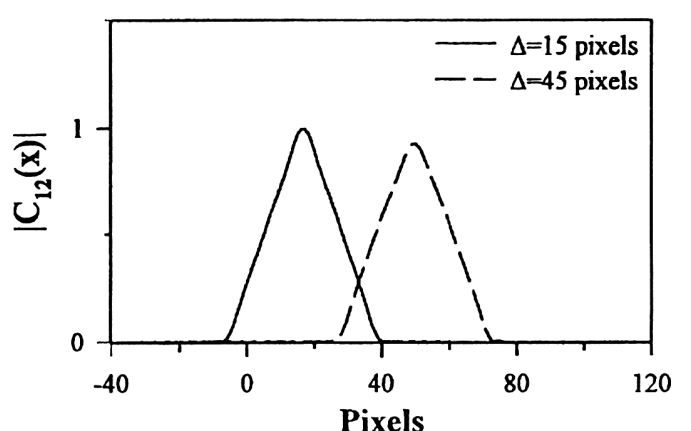

(a)

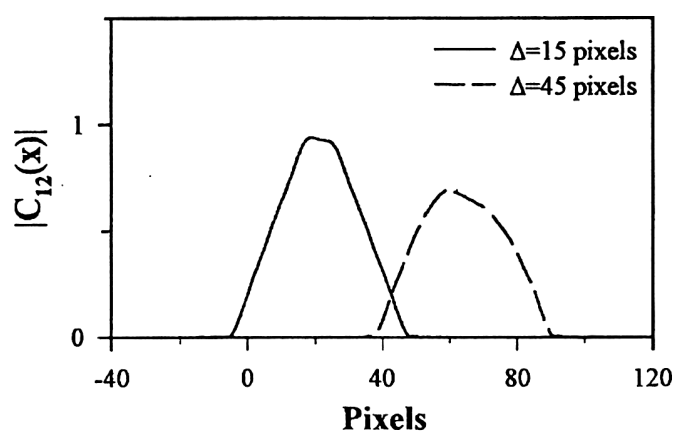

(b)

Fig. 4. (a) Normalized fractional correlation for $q=0.8, r=0.7$, and $p=0.884$ for two different shifts $\Delta$ between $t_{1}\left(x_{0}\right)$ and $t_{2}\left(x_{0}\right)$. (b) Normalized fractional correlation for $q=0.6, r=0.4$, and $p=$ 0.633 for two different shifts $\Delta$ between $t_{1}\left(x_{0}\right)$ and $t_{2}\left(x_{0}\right)$.

where

$$
\begin{aligned}
T_{1}\left(x_{0}{ }^{\prime} ; z_{1}\right) & =t_{1}\left(x_{0}{ }^{\prime}\right) \exp \left(\frac{i \pi x_{0}{ }^{\prime 2}}{\lambda z_{1}}\right), \quad z_{1}=f_{0} \tan \phi_{2} \\
\bar{T}_{2}\left(x_{0}{ }^{\prime} ; z_{2}\right) & =t_{2}\left(\frac{x_{0}{ }^{\prime}}{M}\right) \exp \left[\frac{i \pi}{\lambda z_{2}}\left(\frac{x_{0}{ }^{\prime}}{M}\right)^{2}\right], \quad z_{2}=f_{0} \tan \phi_{3} \\
M & =\frac{\sin \phi_{2}}{\sin \phi_{3}}
\end{aligned}
$$

The fractional correlation as given by Eq. (10) can be thought as the conventional correlation of two functions: $T_{1}\left(x_{0}{ }^{\prime} ; z_{1}\right)$, which is the input signal $t_{1}\left(x_{0}{ }^{\prime}\right)$ multiplied by a zonal phase factor with focal length $z_{1}$, and $\bar{T}_{2}\left(x_{0}{ }^{\prime} ; z_{2}\right)$, which is a scaled version of the product between $t_{2}\left(x_{0}{ }^{\prime}\right)$ and a zonal phase factor with focal length $z_{2}$. Taking into account the analogy between the FRT formalism and the Fresnel diffracted fields, ${ }^{13,14}$ we find that the fractional correlation [Eq. (10)] is closely related to the space-variant Fresnel transform correlator [see Eq. (7) in Ref. 15].

The positional sensitivity of the fractional correlation is illustrated in Figs. 2 and 3 with a simple example: Terms $t_{1}\left(x_{0}\right)$ and $t_{2}\left(x_{0}\right)$ are two identical slits placed at different locations. It can be seen from Figs. 2(b), 2(c), 3(b), and 3(c) how the matching between the zonal phase factors of $T_{1}\left(x_{0}{ }^{\prime} ; z_{1}\right)$ and

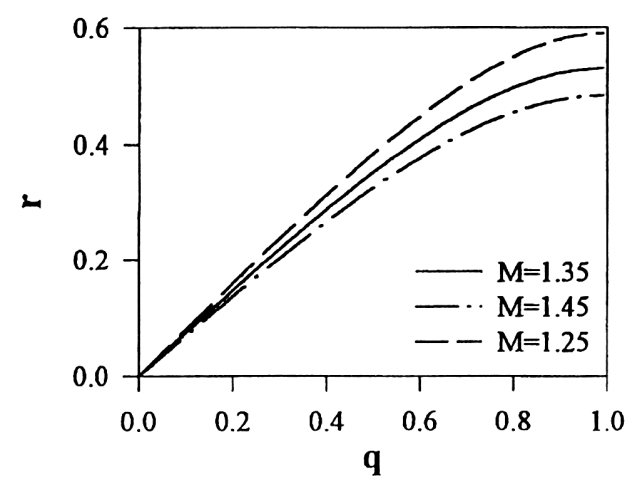

(a)

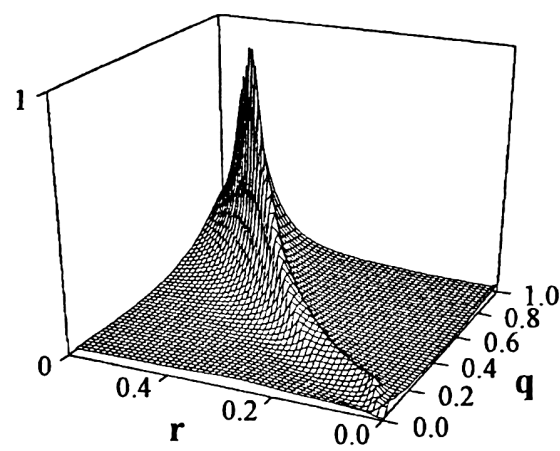

(b)

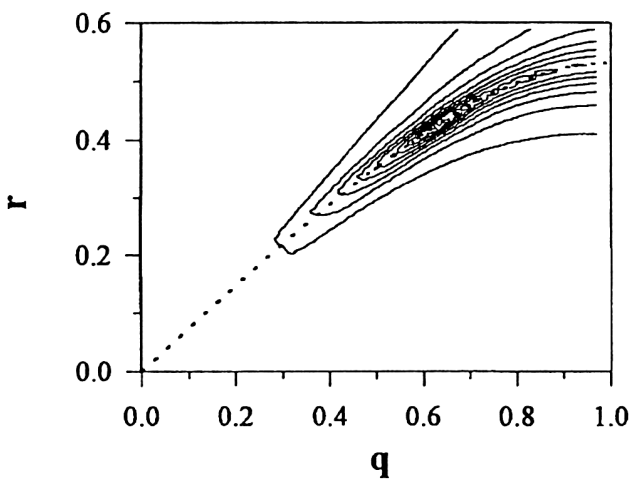

(c)

Fig. 5. (a) Curves showing the set of orders $\left(q_{0}, r_{0}\right)$ that satisfy Eq. (12), i.e., $\sin \left[q_{0}(\pi / 2)\right]=M \sin \left[r_{0}(\pi / 2)\right]$, for three different values of $M$. (b) Contour display of $\alpha^{(p, q, r)}$ for the case of $M=1.35$. (c) Level curves corresponding to the contour plot of (b). The dotted curve represents the theoretical curve of (a) for $m=1.35$.

$\bar{T}_{2}\left(x_{0}{ }^{\prime} ; z_{2}\right)$ depends on the selected values of $p, q$, and $r$.

In Fig. 4, the display of $\left|C_{12}^{(p, q, r)}(x)\right|$ is shown for two different displacements $\Delta_{1}$ and $\Delta_{2}$ between the slit functions $t_{1}\left(x_{0}{ }^{\prime}\right)$ and $t_{2}\left(x_{0}{ }^{\prime}\right)$, along with two different sets of $p, q$, and $r$. As can be seen from these results, the correlation peak diminishes for increasing values of $\Delta$. In addition, this effect becomes more noticeable for decreasing values of $q$ and $r$, as should be expected.

The fact that the fractional correlation becomes a conventional correlation between two functions, $T_{1}(x)$ and $\bar{T}_{2}(x)$, related to the original signals $t_{1}\left(x_{0}\right)$ and $t_{2}\left(x_{0}\right)$, respectively, through a combined phase- 


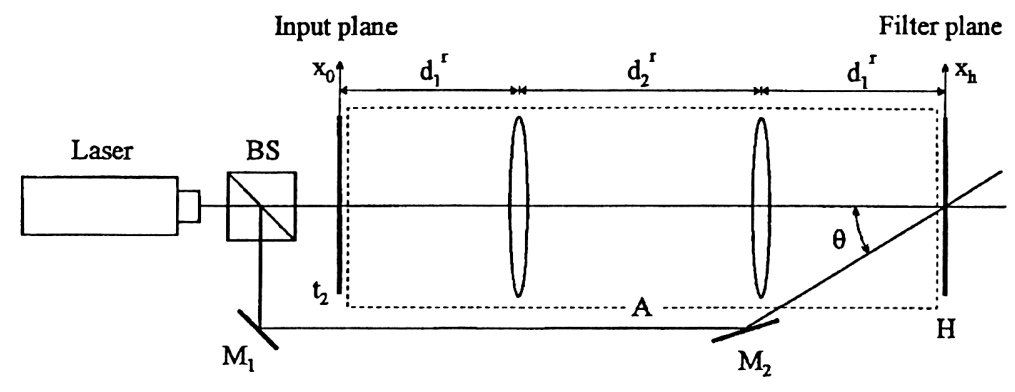

(a)

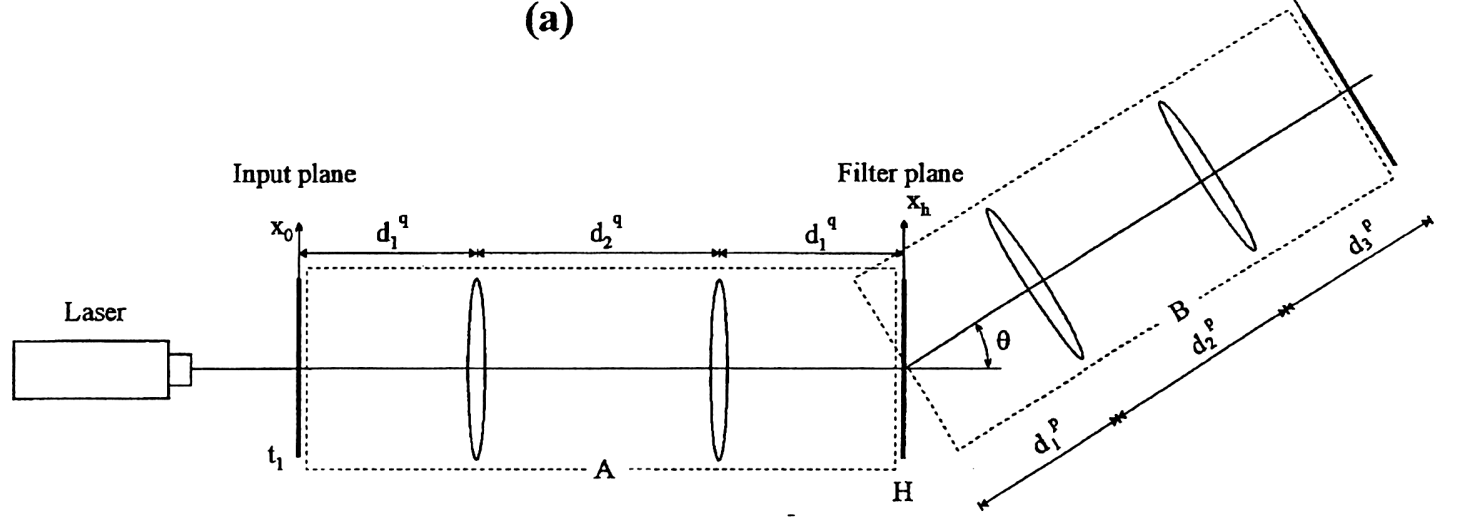

(b)

Fig. 6. Scheme of the optical device employed to achieve (a) the filter $H$, which stores the $r$ th-order FRT of $t_{2}\left(x_{0}, y_{0}\right)$, and (b) the fractional correlation $C_{12}^{(p, q, r)}(x, y)$ at the output plane. $\mathrm{M}$, mirror; BS, beam splitter.

scaling transform [given by Eqs. (11)] suggests how a recognition filter insensitive to scale variations of the input object can be implemented by use of this approach. For two functions $t_{1}\left(x_{0}\right)$ and $t_{2}\left(x_{0}\right)$ that are placed in the same position and differ by only a certain scale factor, several sets of values $\left(p_{0}, q_{0}, r_{0}\right)$ exist for which the fractional correlation $C_{12}^{(p, q, r)}(x)$ becomes the conventional cross correlation of two functions having identical amplitude distributions but slightly different phase factors. This particular set of values $\left(p_{0}, q_{0}, r_{0}\right)$ is illustrated in Fig. 5(a) for three different relative magnifications between $t_{1}\left(x_{0}\right)$ and $t_{2}\left(x_{0}\right)$. To analyze the behavior of the fractional correlation we define a correlation parameter as

$$
\alpha^{(p, q, r)}=\left[\frac{\int\left|C_{12}^{(p, q, r)}(x)-C_{11}^{(-1,1,1)}(x)\right| \mathrm{d} x}{\int C_{11}^{(-1,1,1)}(x) \mathrm{d} x}\right]^{-1},
$$

i.e., it measures the deviation of the fractional correlation between two objects of the same shape but with different magnifications from a conventional autocorrelation of one of these objects for the selected value of the relative magnification $M$ [as given by Eq. (12)].

In Figs. 5(b) and 5(c) the numerical calculation of $\alpha^{(p, q, r)}$ is shown for two slits with a relative magnifi= cation of $M=1.35$. It can clearly be seen that the maxima of $\alpha^{(p, q, r)}$ lie on a curve in the domain of the fractional orders $q$ and $r$; the curve coincides with that previously illustrated in Fig. 5(a) for the case $M=1.35$. However, it can be observed that $\alpha^{(p, q, r)}$ diminishes for decreasing values of $q$ and $r$, a fact that is related to the above-mentioned phase mismatch between $T_{1}(x)$ and $\bar{T}_{2}(x)$. In Section 3 we illustrate the developed analysis with some experimental verifications. Therefore, an optical setup that performs the fractional correlation in a flexible way for varying values of $p, q$, and $r$ could be used to detect objects with magnifications different from that stored in the filter. Recently, Lohmann et al. ${ }^{16}$ developed a similar analysis to that carried out for obtaining Eq. (7) in connection with the space-variance properties of the fractional correlation for three particular cases.

\section{Experimental Results}

The scheme of the optical setup employed to perform the fractional-correlation operations is shown in Fig. 6. A holographic filter $H$ stores the FRT of order $r$ associated with the transparency $t_{1}\left(x_{0}, y_{0}\right)$ by use of optical system $A$ (with distances adequate to the value of $r$ ) and a reference light beam. The whole system $A-B$ becomes a modified $4 f$ configuration. In the first step, subsystem $A$ performs the $q$ th-order FRT of $t_{2}\left(x_{0}, y_{0}\right)$ in the plane containing filter $H$, where the distances $d_{1}^{q}$ and $d_{2}^{q}$ are selected to be $d_{1}{ }^{q}$ $=f \tan (\pi q / 4)+f$ and $d_{2}{ }^{q}=f \sin (\pi q / 2)+2 f$, respectively. Behind the filter $H$ subsystem $B$ carries out the $p$ th-order FRT of the amplitude transmitted by $H$, with distances $d_{1}{ }^{p}=f \tan (\pi p / 4)+f$ and $d_{2}{ }^{p}=f$ 


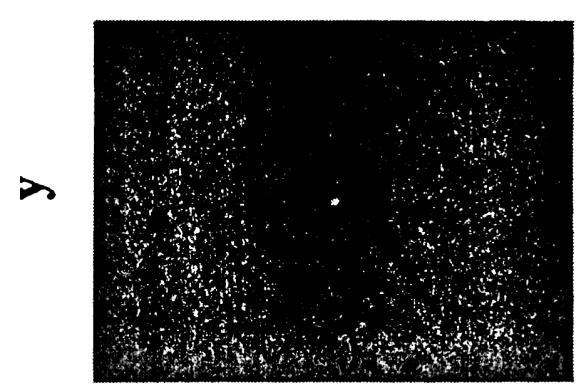

$\mathbf{X}$

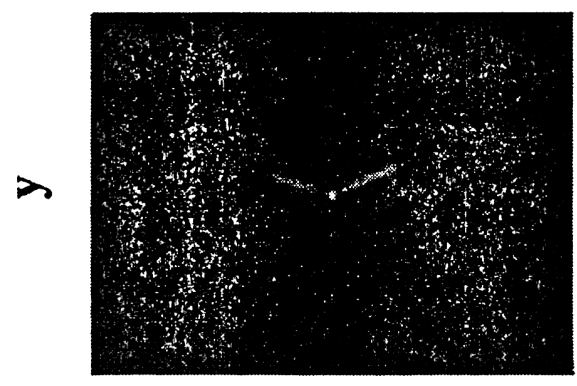

$\mathbf{x}$

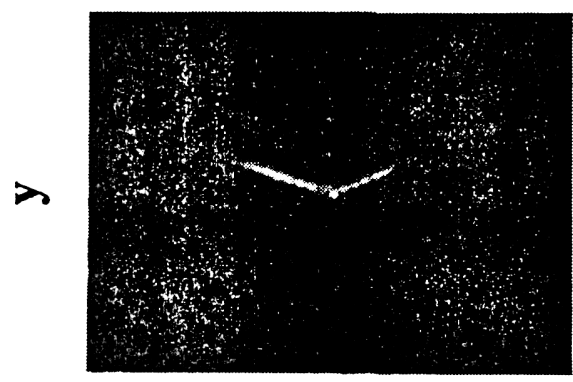

$\mathbf{x}$

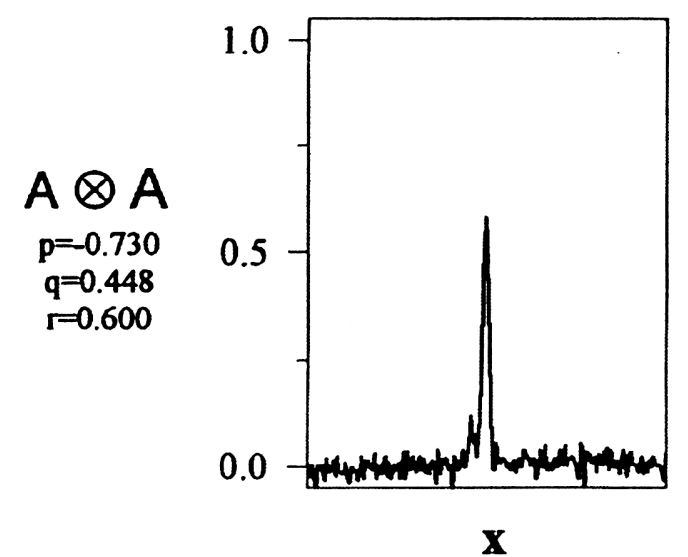

(a)

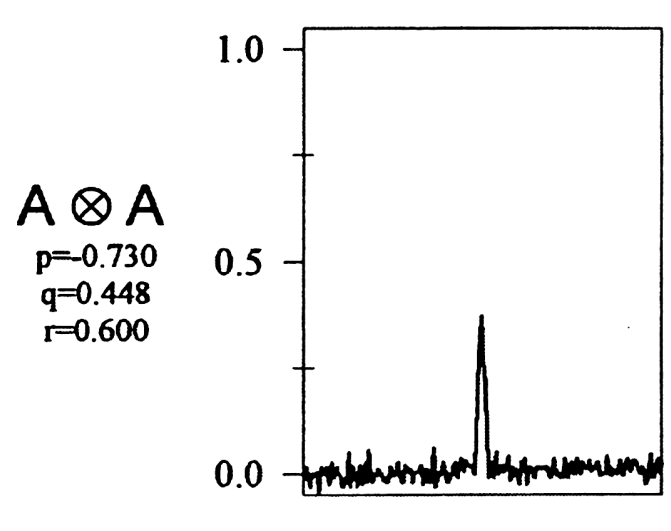

(b)

$\mathbf{x}$

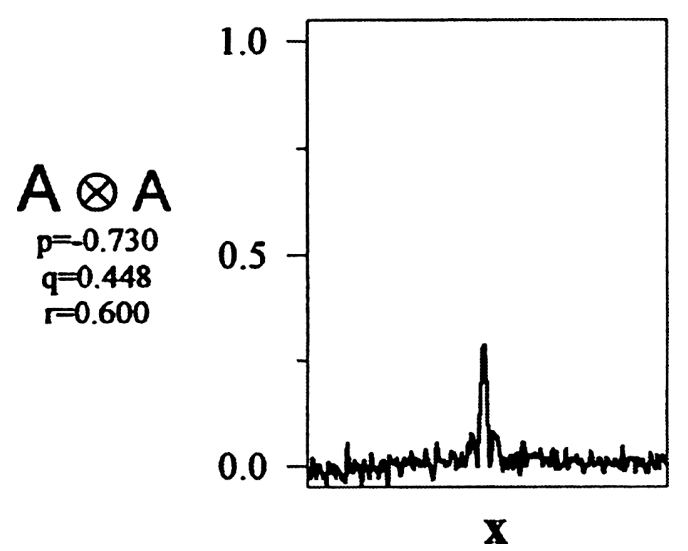

(c)

Fig. 7. Fractional correlation between two characters, A, differing by three relative magnifications: (a) $M=0.80$, (b) $M=1.00$, and (c) $M=1.20$. Since the fractional order were chosen to be $p=-0.730, q=0.448$, and $r=0.600$ [from Eq. (12)], a magnification of $M=0.80$ results, and so the maximum correlation peak appears in (a).

$\sin (\pi p / 2)+2 f$. In this arrangement a fractional correlation $C_{12}^{(p, q, r)}(x, y)$ with arbitrary orders can be obtained by variation of the distances $d_{1}^{q}, d_{2}^{q}, d_{1}^{p}$, and $d_{2}{ }^{P}$. In addition, the FRT's involved have the same parameter $f_{0}$ [see Ref. 17]. Thus, the correlator can easily be adapted to a given object magnif- cation by variation of the orders $q$ and $r$ to select an adequate value of $M$ [Eq. (12)]. A sharp correlation peak is obtained at the output plane of the optical system whenever the objects $t_{1}\left(x_{0}, y_{0}\right)$ and $t_{2}\left(x_{0}, y_{0}\right)$ differ by this scaling factor $M$.

In Figs. 7-9 the fractional correlations between two 


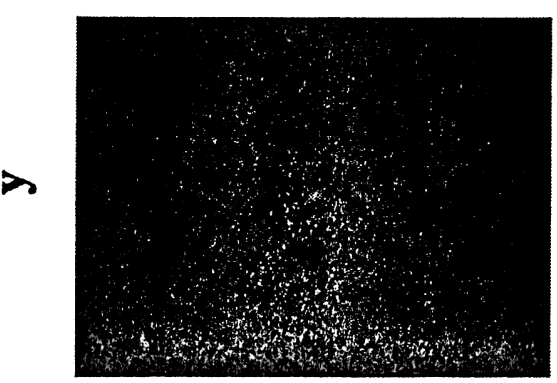

$\mathbf{X}$

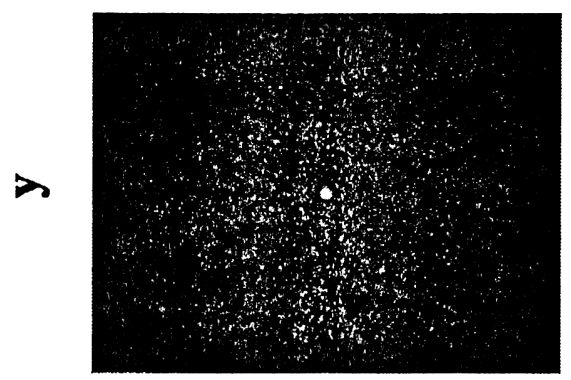

$\mathbf{x}$

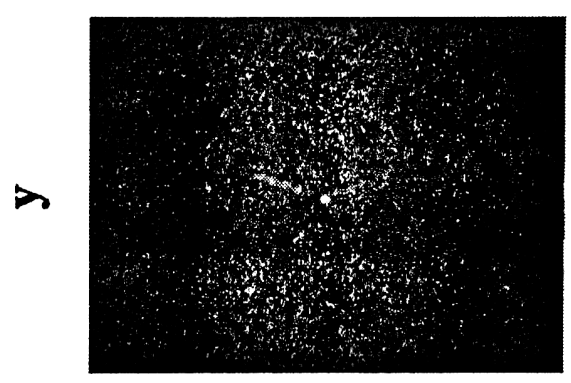

$\mathbf{X}$

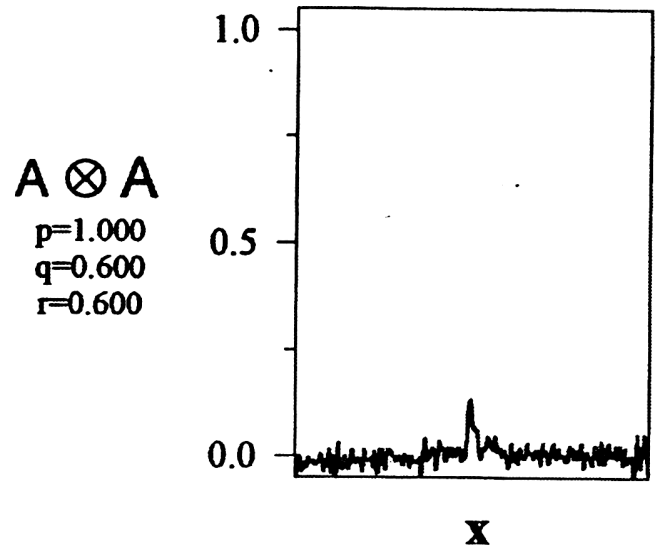

(a)

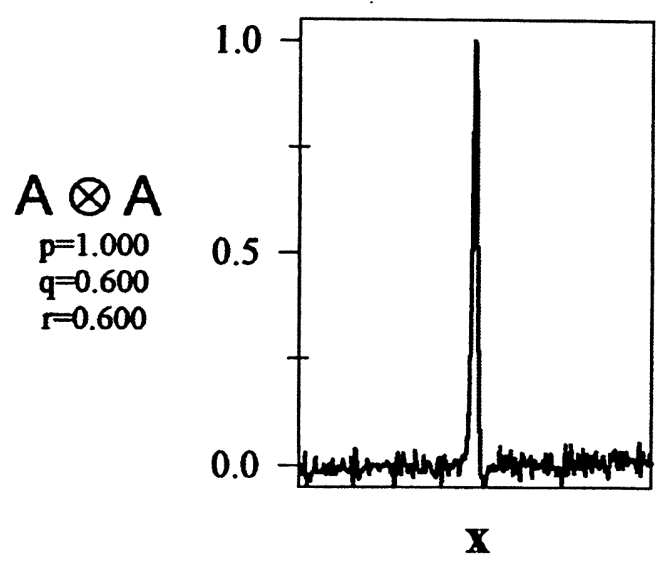

(b)

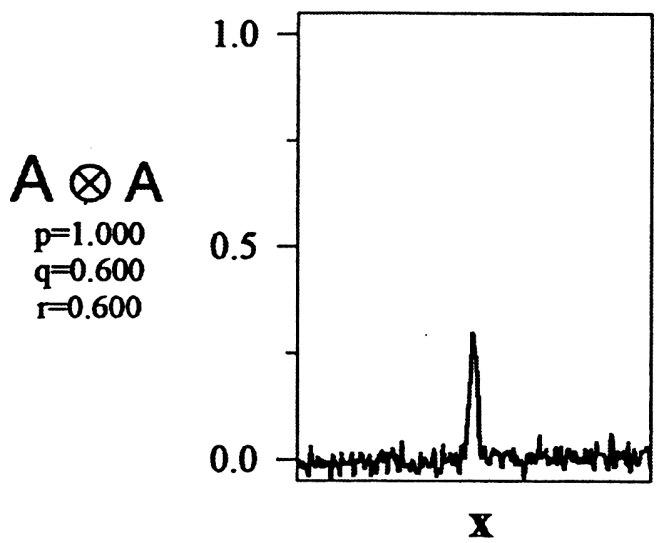

(c)

Fig. 8. Fractional correlation between two characters, A, differing by three relative magnifications: (a) $M=0.80$, (b) $M=1.00$, and (c) $M \equiv 1.20$. Since the fractional orders were chosen to be $p=1.000, q=0.600$, and $r=0.600$ [from Eq. (12)], a magnification of $M=1.00$ results, and so the maximum correlation peak appears in (b).

identical characters with different magnifications are displayed for three sets of $(p, q, r)$. In each of these figures the maximum of the fractional correlation is obtained for the particular character relative magnification that satisfies Eq. (12). In Table 1 the values of the several distances employed are indicated.
Next, we slightly modify optical device $A$ [Fig. $6(a)]$ to record a multiple holographic filter $H$ that stores several FRT's of the same object with different fractional orders $r$. Each recording is performed with a variation of the incident angle $\theta$ of the reference light beam. Now the optical correla- 


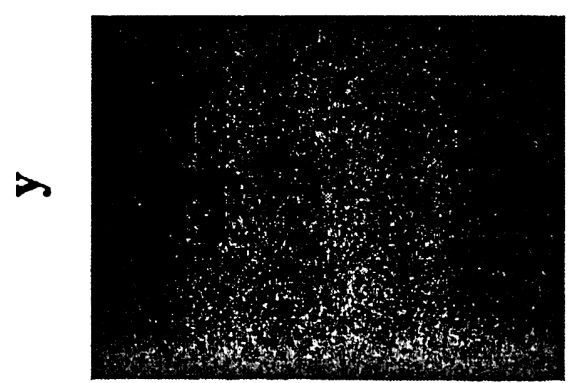

$\mathbf{X}$

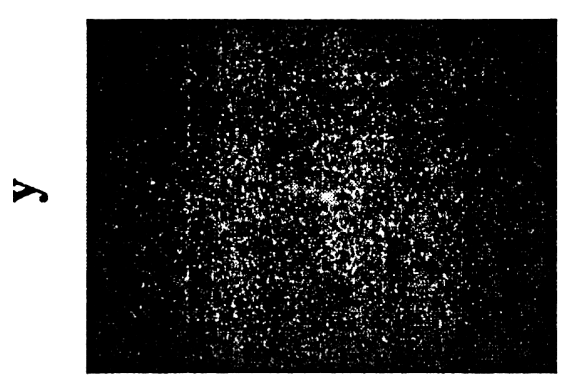

$\mathbf{x}$

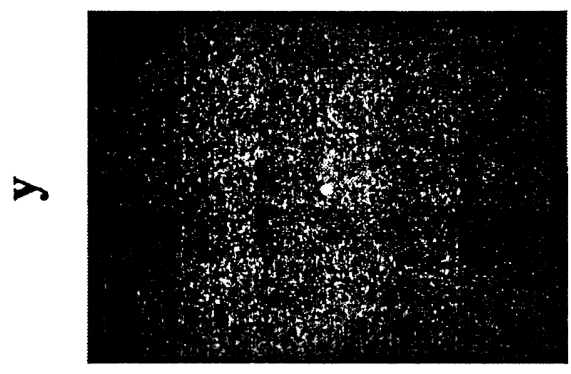

$\mathbf{x}$

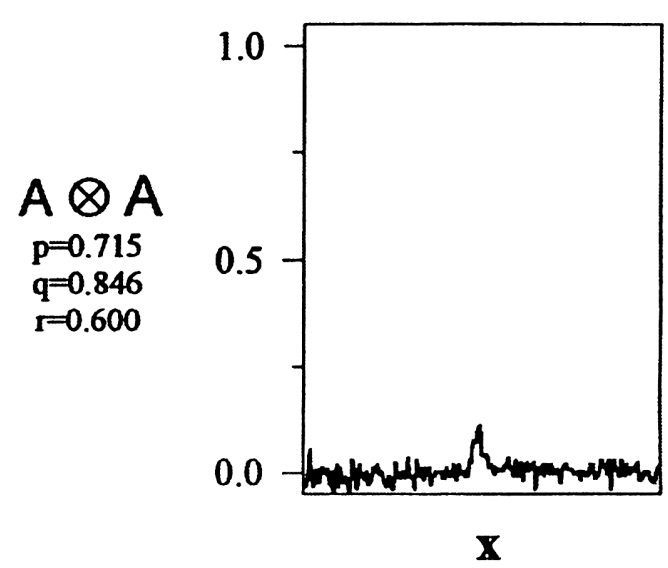

(a)

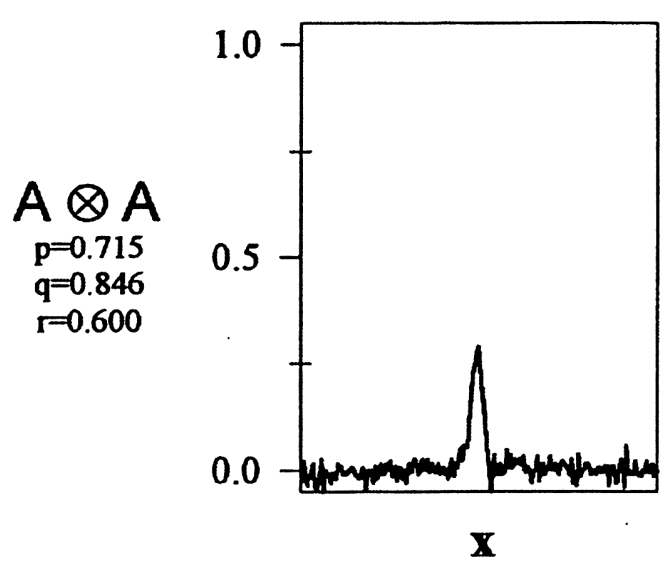

(b)

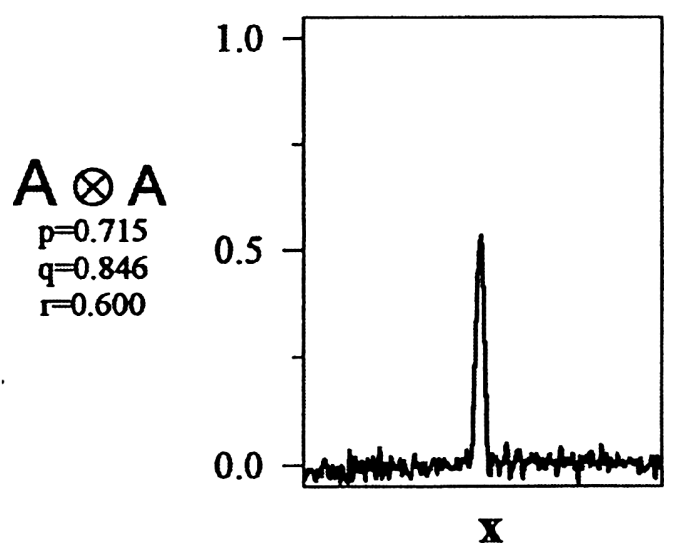

(c)

Fig. 9. Fractional correlation between two characters, A, differing by three relative magnifications: (a) $M=0.80$, (b) $M=1.00$, and (c) $M=1.20$. Since the fractional order were chosen to be $p=0.715, q \equiv 0.846$, and $r=0.600$ [from Eq. (12)], a magnification of $M=1.20$ results, and so the maximum correlation peak appears in (c).

tor [Fig. 6(b)] works in a rather different way: As the distances $d_{1}{ }^{q}$ and $d_{2}{ }^{q}$ remain fixed, the distances $d_{1}{ }^{p}$ and $d_{2}{ }^{p}$ should be selected so as to satisfy the requirements for $(p, q, r)$ established by Eq. (6). This means that a sharp correlation peak appears at the location associated with the angle $\theta$ for which the order $r$ (of the FRT stored in the filter) and the order $q$ satisfy Eq. (12). Thus the filter recognizes, at this particular location $\theta$ (or spatial coordinate $x$ of the output plane), objects with a scale difference given by this value of $M$.

In Fig. 10 we show the results obtained from a 
Table 1. Values of the Distance $d$ Employed in the Experimental Setup of Fig. 6

\begin{tabular}{|c|c|c|c|c|c|c|c|c|c|}
\hline \multirow[b]{3}{*}{ Distance } & \multicolumn{9}{|c|}{ Magnification } \\
\hline & \multicolumn{3}{|c|}{$M=1.0$} & \multicolumn{3}{|c|}{$M=0.8$} & \multicolumn{3}{|c|}{$M=1.2$} \\
\hline & $p=1.000$ & $q=0.600$ & $r=0.600$ & $p=-0.730$ & $q=0.448$ & $r=0.600$ & $p=0.715$ & $q=0.846$ & $r=0.600$ \\
\hline$(m m)$ & 200 & 150.9 & 150.9 & 35.4 & 136.7 & 150.9 & 162.9 & 178.3 & 150.9 \\
\hline$d_{2}(m m)$ & 300 & 280.9 & 280.9 & 108.8 & 264.7 & 280.9 & 290.2 & 297.1 & 280.9 \\
\hline
\end{tabular}
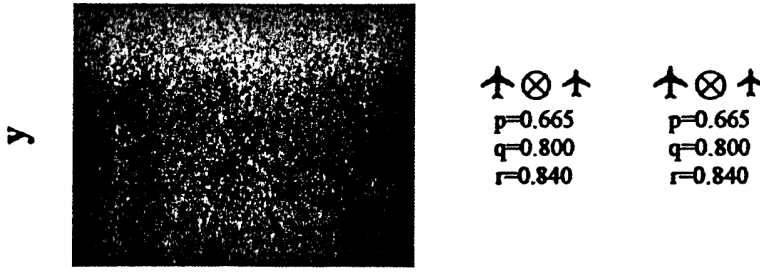

$\mathbf{X}$

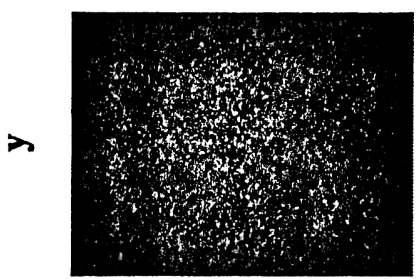

$\mathbf{X}$

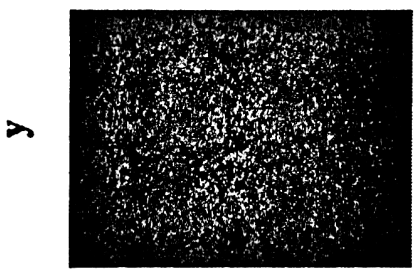

$\mathbf{X}$

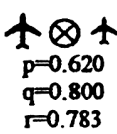

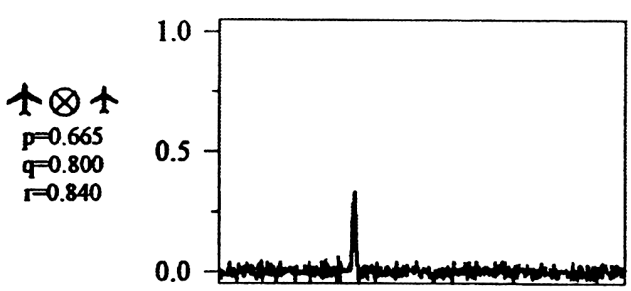

(a)

$\mathbf{x}$
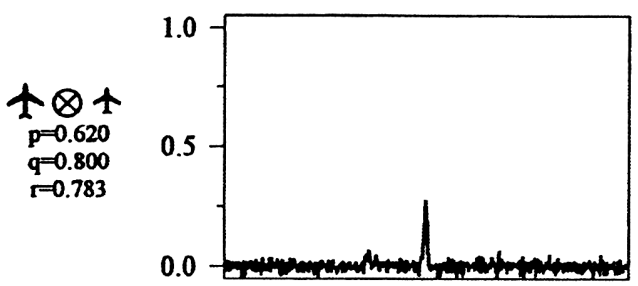

$\mathbf{x}$

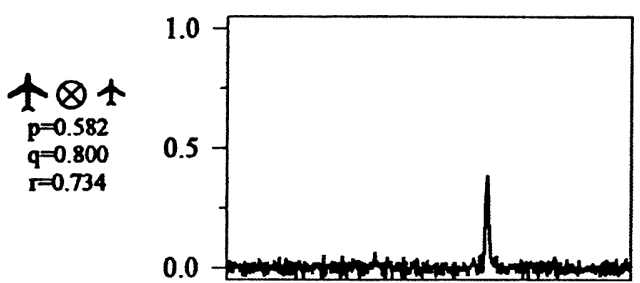

(c)

Fig. 10. Intensity distribution obtained at the output plane of the fractional correlator illustrated by Fig. 6 for a multiple-recorded filter $H$ that stores three FRT's of an airplane-shaped object $t_{2}$ with the orders $r=0.840, r=0.783$, and $r=-0.734$. Depending on the magnification $M$ of the input object $t_{1}$, the maximum correlation peak is detected at different locations: (a) $M=1.10$, (b) $M=-1.15$, and (c) $M=-1.20$.

holographic filter that stores three different FRT's of the same object. Figure 10(a) shows the distances $d_{1}{ }^{p}$ and $d_{2}{ }^{p}$ when they are chosen to yield a value of $M=1.10$. Therefore, a sharp correlation peak appears at the location associated with the angle $\theta$ corresponding to the stored FRT of the order $r=0.840$. In Figs. $10(\mathrm{~b})$ and $10(\mathrm{c})$, results for the same procedure are shown for achieving $M=1.15$ and $M=1.20$, respectively. The correlation maxima now appear at the locations corresponding to the stored FRT's of orders $r=0.783$ and $r=0.734$, respectively. In this way, depending on which magnification of the object $t_{1}\left(x_{0}, y_{0}\right)$ is present, the correlation peak is found at different locations in the output plane. Of course, the range of object scales that can be recognized by the filter is limited by the maximum number of FRT's that can be stored in the holographic medium.

\section{Conclusions}

In this paper we have analyzed the fractional correlation in connection with the space-variance properties of the FRT. It was found that, for specific values of the fractional orders, the fractional correlation becomes insensitive to scale changes of the input object. An optical configuration was proposed for obtaining the fractional correlation in a flexible way. Thus, the orders of the FRT's involved in the fractionalcorrelation operation can easily be varied to match the filter to any required object magnification. 
Financial support from the Consejo Nacional de Investigaciones Científicas y Técnicas (Argentina) is gratefully acknowledged.

\section{References}

1. A. Vander Lugt, "Signal detection by complex spatial filtering," IEEE Trans. Inf. Theory IT-10, 139-140 (1964).

2. C. S. Weaver and J. W. Goodman, "A technique for optically convolving two functions," Appl. Opt. 5, 1248-1249 (1966).

3. D. Mendlovic, H. M. Ozaktas, and A. W. Lohmann, "Fractional correlation," Appl. Opt. 34, 303-309 (1995).

4. D. Mendlovic, Y. Bitran, R. G. Dorsch, and A. W. Lohmann, "Optical fractional correlation: experimental results," J. Opt. Soc. Am. A 12, 1665-1670 (1995).

5. V. Namias, "The fractional Fourier transform and its applications in quantum mechanics," J. Inst. Math. Appl. 25, 241-265 (1980).

6. H. M. Ozaktas and D. Mendlovic, "Fourier transforms of fractional order and their optical implementation," Opt. Commun. 101, 163-169 (1993).

7. D. Mendlovic and H. M. Ozaktas, "Fractional Fourier transforms and their optical implementation: I," J. Opt. Soc. Am. A 10, 1875-1881 (1993).

8. H. M. Ozaktas and D. Mendlovic, "Fractional Fourier transforms and their optical implementation: II," J. Opt. Soc. Am.
A 10, 2522-2531 (1993).

9. A. W. Lohmann, "Tmage rotation, Wigner rotation, and the fractional Fourier transform," J. Opt. Soc. Am. A 10, 21812186 (1993).

10. D. Mendlovic, H. M. Ozaktas, and A. W. Lohmann, "Gradedindex fiber, Wigner distribution functions, and the fractional Fourier transform," Appl. Opt. 33, 6188-6193 (1994).

11. A. Papoulis, "Ambiguity function in Fourier optics," J. Opt. Soc. Am. 64, 779-788 (1974)

12. S. Y. Lee and H. H. Szu, "Fractional Fourier transform, wavelet transform, and adaptive neural networks," Opt. Eng. 33, 2326-2329 (1994).

13. P. Pellat-Finet, "Fresnel diffraction and the fractional order Fourier transform," Opt. Lett. 19, 1388-1390 (1994).

14. S. Granieri, O. Trabocchi, and E. E. Sicre, "Fractional Fourier transform applied to spatial filtering in the Fresnel domain," Opt. Commun. 119, 275-278 (1995).

15. J. A. Davis, D. M. Cottrell, N. Nestorovic, and S. M. Highnote, "Space-variant Fresnel transform optical correlator," Appl. Opt. 31, 6889-6893 (1992).

16. A. W. Lohmann, Z. Zalevsky, and D. Mendlovic, "Synthesis of pattern recognition filters for fractional Fourier processing," Opt. Commun. 128, 199-204 (1996).

17. A. W. Lohmann, "A fake zoom lens for fractional Fourier experiments," Opt. Commun. 115, 437-443 (1995). 